\title{
Constant strain rate compression of bovine cortical bone on the Split-Hopkinson Pressure Bar
}

\author{
A. Bekker ${ }^{\mathrm{a}, \mathrm{b}}$, ,T.J.Cloete ${ }^{\mathrm{a},}$ A. Chinsamy-Turan ${ }^{\mathrm{c}}, \mathrm{G}$. N.Nurick ${ }^{\mathrm{a}}$, S.Kok ${ }^{\mathrm{a}, \mathrm{d}}$
}

a Blast Impact and Survivability Research Unit, Department of Mechanical Engineering, University of Cape Town, Private Bag X3, Rondebosch 7701, South A fric a b Department of Mechanical and Mechatronic Engineering, University of Stellenbosch, Private Bag X1, Matieland 7602, South Africa c Department Biological Sciences, Private Bag X3, Rondebosch 7701, South Africa

d Department of Mechanical and Aeronautical Engineering, University of Pretoria, Private Bag X20, Hatfield, Pretoria 0028, South Africa

A b s t r a c t

Cortical bone is a visco-elastic material which implies that strain rate will affect its response. Although the Split-Hopkinson Pressure Bar is an accepted technique for determining the dynamic compressive properties of cortical bone it has been shown that the strain rate of compression does not remain constant throughout the duration of a classical experiment with a uniform striker. This raises concerns as to the measurement of smeared responses. This paper presents a shaped striker technique whereby the incident pulse can be shaped to attain a constant strain rate experiment for bovine bone. Shaped strikers offer benefits such as reusability and increased test repeatability. A comparison of the stress-strain-strain rate responses attained through classical and constant strain rate experiments shows that the shape of the stress-strain curves from conventional experiments is adversely affected in the portion where the strain rate varies. The dynamic response corridors for the two tests are similar, however the ultimate properties are affected. It is concluded that the strain rate history should be presented with dynamic stress-strain responses since the instantaneous strain rate is a likely contributor to potential constitutive models.

Keywords:

Strain rate effects

Bovine cortical bone

Split-Hopkinson

Pressure bar

Shaped striker

Compression

\section{Introduction}

The primary function of bone is to support the body, thus providing a mechanical basis for movement. The load bearing ability of long bones is ascribed to cortical bone, which is primarily situated in the diaphysis (middle, shaft region) of long bones (such as the tibia and femur) [1]. This bone structure protects vital organs and prevents the excessive deformation of surrounding tissues under the influence of external forces.

The computational simulation of the complex, dynamic interactions of the human body is becoming a useful and cost-effective research and development tool [2]. This is especially true in hazardous environments such as impact, ballistic and blast loading situations where designs are evaluated for their ability to mitigate injury or improve survivability [3]. These computational models require calibrated material models, that are accurate over a large range of strain rates [4].

The fracture and pre-fracture responses of bone for computational representation are determined through mechanical testing. Several investigations on the dynamic and quasi-static behaviour of bone have determined that factors such as material orientation [5-8], the mode

\footnotetext{
* Corresponding author at: Blast Impact and Survivability Research Unit, Department of Mechanical Engineering, University of Cape Town, Private Bag X3, Rondebosch, 7701, South Africa.

E-mail address: annieb@sun.ac.za (A. Bekker).
}

of loading (tension or compression) [9], age [10], gender [11] and bone mineral density [12] influence the fracture and pre-fracture responses of cortical bone. This paper, however, focusses on the ratedependent nature of bone which has been documented by numerous studies over the past fifty years [4,5,13-17].

Hight [3] formulated one of the earliest bone fracture criteria which include a loading-rate dependency. The criterion is based on uni-axial compression tests by McElhaney [13] on the bone from human and bovine femurs at strain rates between $10^{-3} \mathrm{~s}^{-1}$ and $1.5 \times 10^{3} \mathrm{~s}^{-1}$. Research indicates that the strain rate sensitivity of cortical bone is evident in that its final fracture occurs at a higher stress and lower strain with increased loading rate. This behaviour is prevalent in both tension [14] and compression [13] for small changes in both quasi-static and dynamic strain rates [18].

The use of the Split-Hopkinson Pressure Bar (SHPB) [19] is a well documented technique for the investigation of the dynamic properties of cortical bone [5,14-17,20,21]. Tennyson et al. [16] investigated the dynamic characteristics of bovine cortical bone as a function of elapsed post-mortem time. Specimens from the dense posterior section of the femoral mid-shaft were exposed to a wide range of strain rates $\left(10 \mathrm{~s}^{-1}\right.$ to $\left.450 \mathrm{~s}^{-1}\right)$ by direct compression on the SHPB. This study reported that the strain rate varied during the course of each experiment. Tanabe and Kobayashi [5] and Ferreira et al. [15] investigated the compressive response of bovine bone prior to yield. Neither studies reported 
the strain rate histories of their experiments. Ferreira et al. [15] did however mention that the maximum strain rate varied between $368 \mathrm{~s}^{-1}$ and $795 \mathrm{~s}^{-1}$, and the strain rate at fracture between $41 \mathrm{~s}^{-1}$ and $386 \mathrm{~s}^{-1}$.

Adharapurapu et al. [17] compressed bovine bone specimens at various strain rates $\left(10^{-3}\right.$ to $10^{3} \mathrm{~s}^{-1}$ ) in both the longitudinal (parallel to the axis of the bone) and transverse (perpendicular to the axis of the bone) material directions. The researchers observed that it was difficult to achieve constant strain rate dynamic compression of bovine cortical bone samples with SHPB experiments using a uniform striker. Given the evident sensitivity of bone response to strain rate Adharapurapu et al. [17] hypothesised that a varying strain rate during a test could result in the measurement of a smeared response. Adharapurapu et al. [17] succeeded in conducting a constant strain rate experiment by using a pulse shaping technique on the SHPB. This technique involved placing a deformable metal disc on the impact surface of the incident bar, thereby using the work hardening properties of the pulse shaper to increase the slope of the input stress wave. Although Adharapurapu et al. [17] reported that both constant and non-constant strain rate compression experiments were conducted, no comparison was made between the responses measured in the two experiments.

An alternative to sacrificial pulse shapers is the use of non-uniform (or shaped) strikers to manipulate the shape of the incident pulse on the SHPB. This too is a well-known technique that has been used in the testing of brittle materials [22] and in deformation instability studies [23]. Kumar et al. [22] found that if a conical striker impacts a SHPB with its small end first, this will result in an accelerator pulse in the bar that increases in steepness as a function of the cone angle.

The present investigation proposes the use of shaped strikers as an attractive alternative to achieve constant strain rate compression of cortical bone on the SHPB. Furthermore this work aims to establish a comparison of the mechanical properties of bovine cortical bone in longitudinal compression as determined from varying and constant strain rate SHPB experiments.

\section{Materials and methods}

Bovine femurs were purchased from a local butchery after being transported in a refrigeration truck from the abattoir (about $60 \mathrm{~km}$ ). All meat and most of the fibrous tissue were removed when the bones were acquired approximately 3 to 7 days after slaughtering. The femur bones were thawed in water for several days before machining specimens from the mid-diaphysis. In order to study the effect of strain rate on compression, ten cylindrical specimens (diameter $5.99 \pm 0.07 \mathrm{~mm}$, length $5.95 \pm 0.12 \mathrm{~mm}$ ) were turned from the middiaphysis of each of eleven bovine femurs and refrigerated in water until quasi-static and dynamic compression experiments could be performed. The specimens were orientated in the longitudinal direction as shown in Fig. 1.

Excess bone material was dried and preserved for histological examination through the method of thin sectioning as described by Chinsamy and Raath [24]. The histology of the bovine bones was investigated in two planes: transverse sections cut through the bone in a plane that is perpendicular to the bone axis and longitudinal sections which are made in the plane of the bone axis (Fig. 1).

\subsection{Bone compression experiments}

Quasi-static compression tests (at strain rates of $10^{-4} \mathrm{~s}^{-1}$ to $10^{-1} \mathrm{~s}^{-1}$ ) were performed on a conventional screw-driven test machine under displacement control. The SHPB technique [19] was implemented to conduct dynamic compression experiments at two strain rates $\left(2.5 \times 10^{2} \mathrm{~s}^{-1}\right.$ and $\left.10^{3} \mathrm{~s}^{-1}\right)$. Diametrically opposed foil strain gauges ( $2 \mathrm{~mm}, 120 \Omega$ ) were bonded to the midpoint of the Hopkinson bars. The signals were amplified using custom-built amplifiers (gain of 1000 , bandwidth of $100 \mathrm{kHz}$ ) and digitized using a ADLINK PCI-9812 card (10 MHz, 12 bit). The silver steel bars in both experiments were

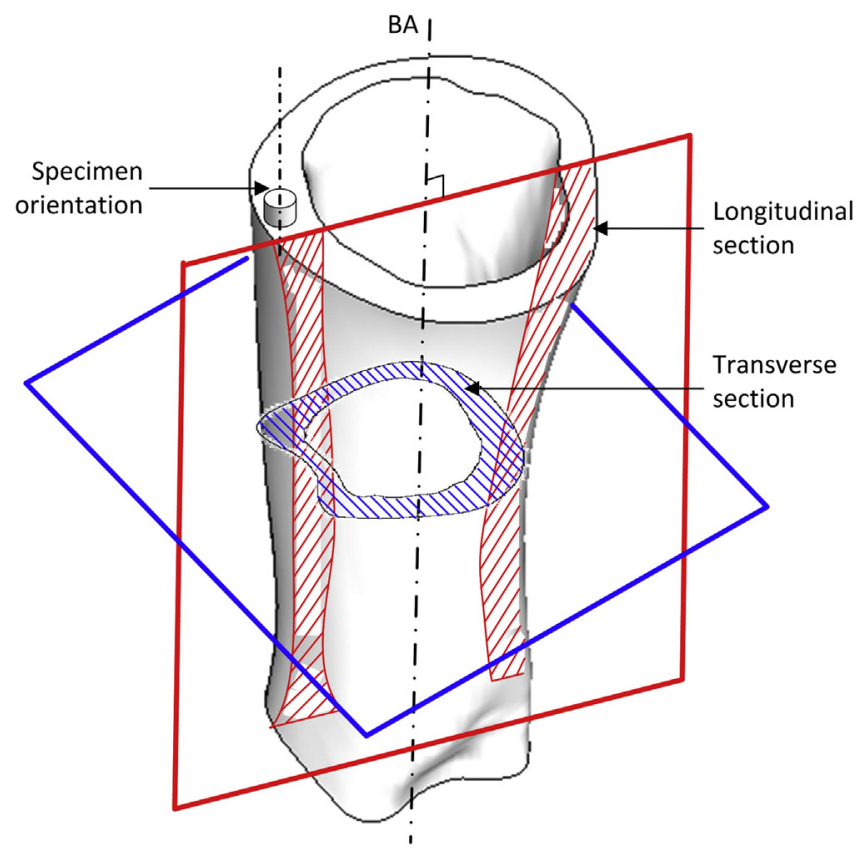

Fig. 1. A diagram indicating the longitudinal orientation of the cylindrical specimens as well as the longitudinal and transverse section planes.

determined to have a density of $7938 \mathrm{~kg} \cdot \mathrm{m}^{-3}$ and a wave propagation speed of $5143 \mathrm{~m} \cdot \mathrm{s}^{-1}$.

Bone compression at $2.5 \times 10^{2} \mathrm{~s}^{-1}$ was achieved with $20 \mathrm{~mm}$ diameter bars with a length of $2 \mathrm{~m}$. A uniform aluminium striker with a diameter of $12 \mathrm{~mm}$ was used in a conventional Split-Hopkinson bar experiment to verify the variation of strain rate during the test. Constant strain rate experiments were conducted using a shaped, 7075-T6 Aluminium striker (Fig. 2) with a length of $550 \mathrm{~mm}$ and end diameters of $19.02 \mathrm{~mm}$ and $10.11 \mathrm{~mm}$ respectively. The striker was fired such that the smaller diameter impacted the incident Hopkinson bar first (Fig. 3) so as to result in an accelerator (ramped) incident pulse.

Bone compression at $10^{3} \mathrm{~s}^{-1}$ was executed on smaller, $12 \mathrm{~mm}$ diameter bars with a length of $1 \mathrm{~m}$. The responses resulting from a $10 \mathrm{~mm}$ uniform diameter steel striker were compared to those of a conical striker of 17-4PH stainless steel with a length of $169 \mathrm{~mm}$ and respective end diameters of $11.8 \mathrm{~mm}$ and $6.52 \mathrm{~mm}$. A photograph of the smaller conical striker is included in Fig. 2. The theory of stress wave propagation in conical bars is more complicated than for uniform bars and there is no simple analytical technique to predict the resulting stress wave shapes. Hence, for this work, a relatively simple one dimensional numerical method, similar to that used by Kumar et al. [22], was implemented to estimate the conical striker dimensions. Typical results from the numerical predictions are presented in Fig. 4. The stress wave predictions for four different conical strikers of the same length are compared. All the strikers are simulated to be impacting at the same speed with the small end first against a Hopkinson bar of $20 \mathrm{~mm}$ diameter. All the strikers have a small diameter of $10 \mathrm{~mm}$ but the large diameters that vary between $10 \mathrm{~mm}$ to $30 \mathrm{~mm}$.

The predicted signals have the required features of a virtually instantaneous stress rise, followed by a gradual increase in stress which ends with a sudden stress deterioration. The undesirable feature of a stress wave tail, which gradually drops to zero, is present if the ratio of the large and small diameters is substantial. The 'tail' is explained as follows: When the striker impacts the input bar, stress waves originate at the impact surfaces. The stress wave in a uniform area striker would run to the end of the striker and back to the interface. At this point the striker will lose contact with the bar if it has a smaller diameter than the bar. This is not the case with a non-uniform striker, impacting with the small end first. The diameter increase across the length implies 


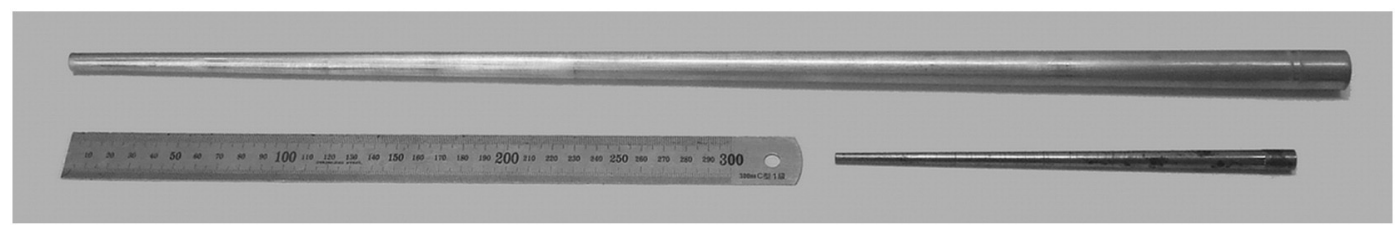

Fig. 2. A photograph of the long 7075-T6 Aluminium and short 17-4PH stainless steel conical strikers.

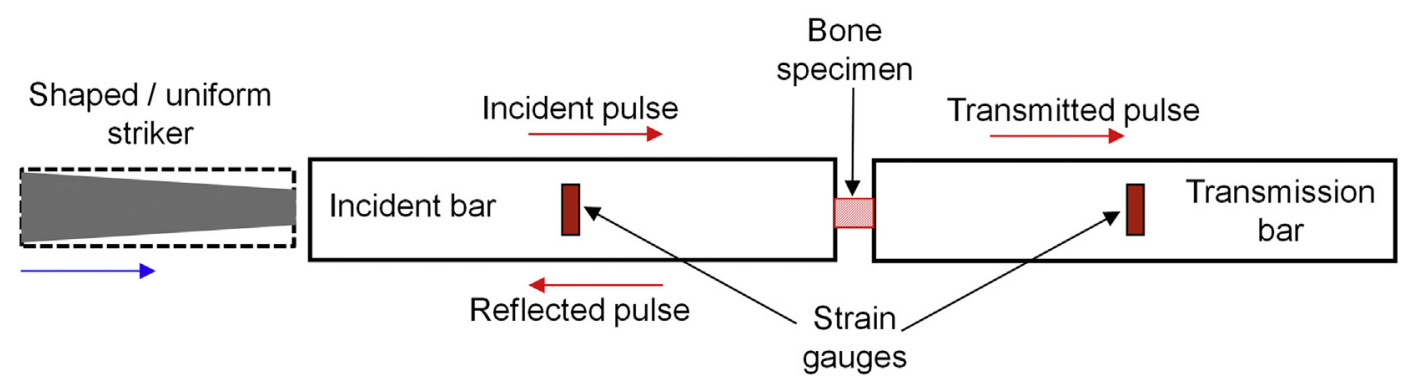

Fig. 3. The layout of the Split-Hopkinson Pressure Bar for dynamic compression testing of bovine cortical bone.

that there is a residual mass that must be decelerated and this accounts for the 'tail'. The 'tail' is undesirable in that it restricts the maximum duration of a test for a given SHPB length. Finally, the simulations indicate that the stress drops to zero, which is the release point where the striker and bar move apart since the impact interface cannot support a tensile stress. The simulated stresses can become negative because a contact release mechanism has not been included in the model. The initial stress rise and the duration of the stress ramp are the same for all the strikers, which indicates that these features depend only on the size of the small diameter and the striker length respectively. Both of the features can be predicted using simple stress wave theory. The gradient of the subsequent stress ramp and the duration of the tail increase with an increase in the size of the large diameter.

\section{Results}

\subsection{Microstructure}

The transverse sections of the bovine femur diaphyses reveal that the histology is arranged in roughly concentric rings between the inner and outer bone surfaces. The transverse microstructure is predominated by two arrangements namely, plexiform microstructure and woven bone. A micrograph showing the arrangement of the vascular networks within a woven fibred bone matrix is magnified in Fig. $5 \mathrm{a}$. This shows a concentration of longitudinally aligned primary osteons within the woven bone matrix. A canal that runs down the centre of each osteon contains blood vessels, nerves and other cellular structures [25]. The mineral matrix between the central canal and outer boundary of both primary and secondary osteons is arranged in concentric lamellae. This is evident from the concentric arrangement of the osteocytes and the texture of the bone. Sasaki et al. [26] suggest a possible reason for the visco-elasticity of bone to be the collagen fibres that comprise these lamellae. Fig. 5b shows the magnification of a typical plexiform microstructure with predominantly circumferential canals with radial anastomosis (branching network). Plexiform bone is primarily found in large rapidly growing animals such as bovine or ovine [28]. Mineral buds grow first perpendicular and then parallel to the outer bone surface, which produces the brick like structure characteristic of plexiform bone.

Longitudinal sections of the bovine femur diaphyses presented with one of three microstructural arrangements: laminar, reticular or plexiform. Investigation of the plexiform and laminar (Fig. 6a) cortical bone sections display a vascular porosity that is more or less aligned with the orientation of the bone axis. In contrast the vasculature of the

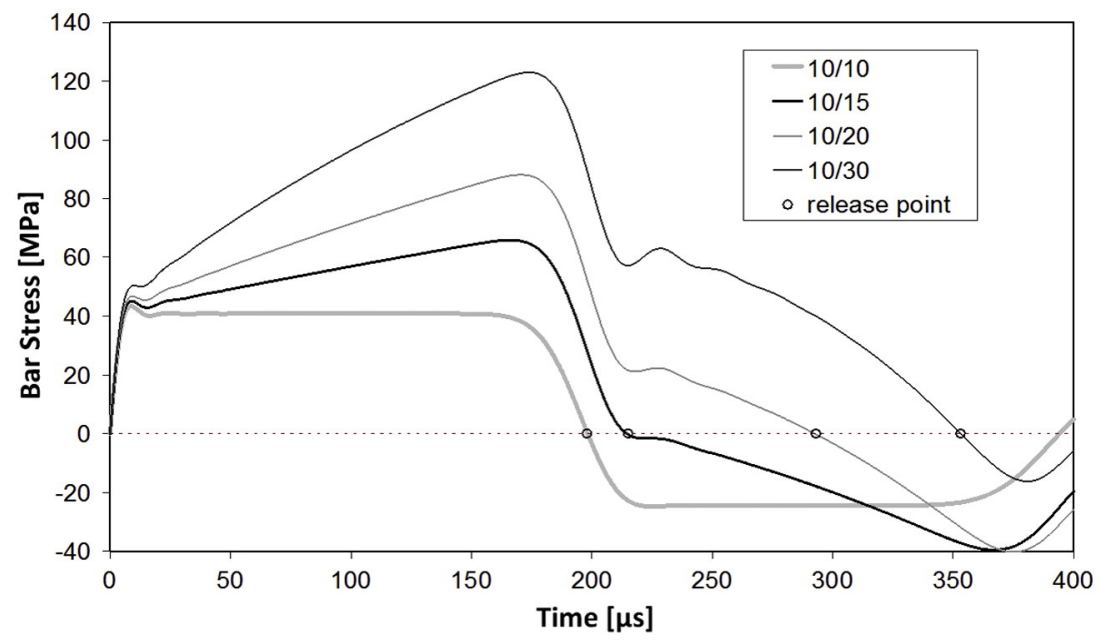

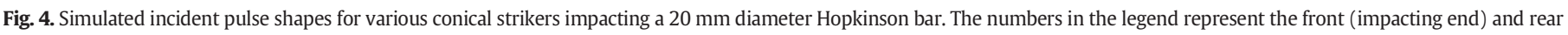
cone diameters. 
a

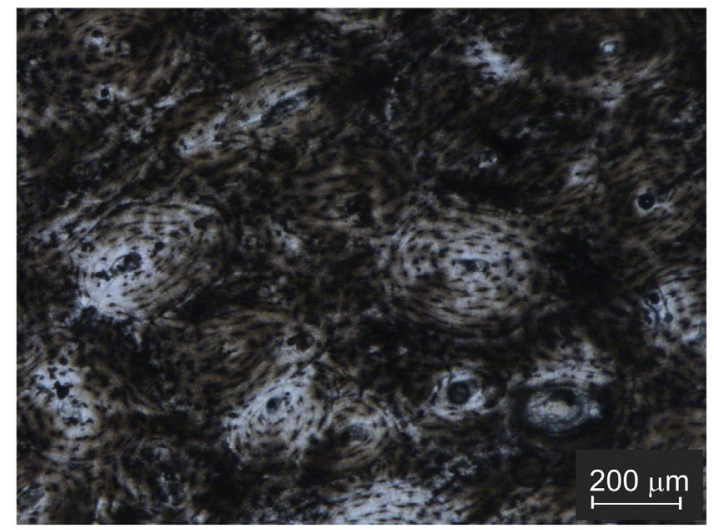

b

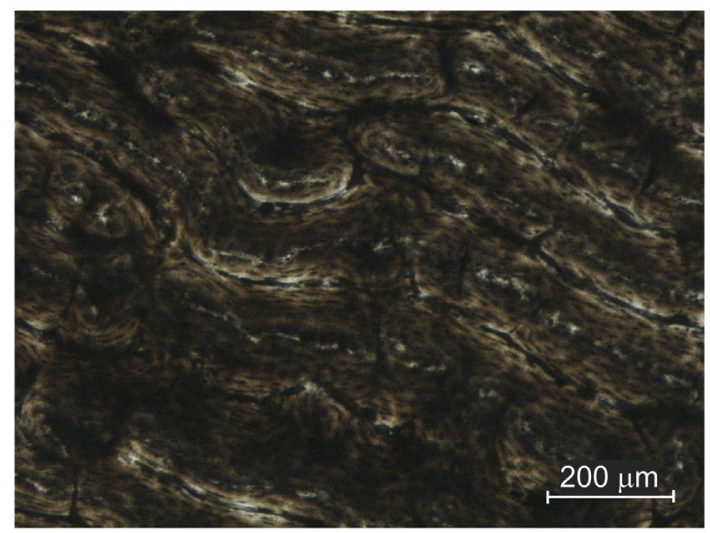

Fig. 5. Micrographs of cortical bone histology in transverse sections of bovine bone diaphyses show (a) woven bone with a concentration of longitudinally aligned primary osteons and (b) plexiform bone where the organisation of the vascular canals forms circumferential and radial networks.

reticular arrangement appears unorganised in the bone matrix. Fig. $6 \mathrm{~b}$ presents a micrograph of reticular microstructure as viewed through a polarizing filter. The filter causes all the apatite crystals with the same orientation to be displayed in the same colour. It can be seen that the apatite crystals in the reticular structure do not have a uniform orientation, but rather an amorphous arrangement.

This investigation identified a variation of microstructural arrangements in bovine cortical bone specimens which are consistent with literature $[17,29]$. To date, research on micromechanical models [30] suggests that these arrangements influence the properties of bone, such as its mechanical stiffness. The effects of different microstructural arrangements on the rate-dependent properties of meso-scale bovine cortical bone models are not presently known. The experimental techniques suggested in this study could enable an investigation into such factors.

\subsection{Bone compression experiments}

The averaged stress-strain curves as determined from the quasistatic and dynamic compression experiments are presented in Fig. 7. It is clear that bone responds to quasi-static and dynamic compression in two distinct corridors. The dynamic response corridor is significantly more stiff than the quasi-static one. This implies that if a bone specimen is deformed to a certain level, the stress that develops in the material would be much higher if the load was dynamic, than if it were quasistatic. Experiments show that bovine bone fractures at a higher stress with an increasing strain rate as shown in Table 1. This trend of increasing failure stress remains consistent throughout the quasi-static and dynamic strain rate regimes. The final strain at fracture increased a
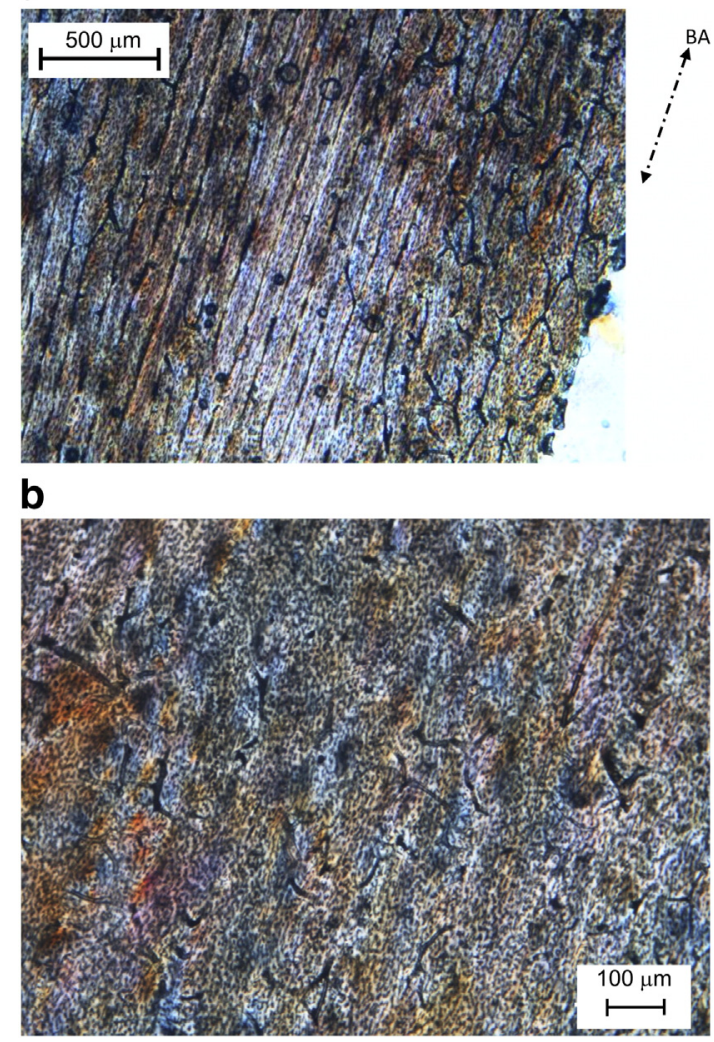

Fig. 6. Micrographs of longitudinal bone sections. BA denotes the direction of the longitudinal bone axis. (a) A polarized image of a specimen with a transition between laminar and reticular bone structure and (b) a reticular microstructure which shows the non-uniform 3-D organisation of channels and lacunae through a polarization filter.

consistently from $\dot{\varepsilon}=10^{-4} \mathrm{~s}^{-1}$ to $\dot{\varepsilon}=10^{-1} \mathrm{~s}^{-1}$. However, the opposite is true for the transition between quasi-static, where the ultimate strains were about $6.2 \%$ as opposed to dynamic strain rates where the ultimate strain is about $2.4 \%$. The response is more brittle in that specimens fracture at a significantly smaller ultimate strain when they are compressed dynamically. There is a significant difference in bone response within the range of strain rates between $\dot{\varepsilon}=0.1 \mathrm{~s}^{-1}$ and $\dot{\varepsilon}=2.5 \times 10^{2} \mathrm{~s}^{-1}$. This large difference suggests that bovine cortical bone responds in a highly rate-sensitive manner when compressed at the afore-mentioned strain rates. Unfortunately this regime coincides

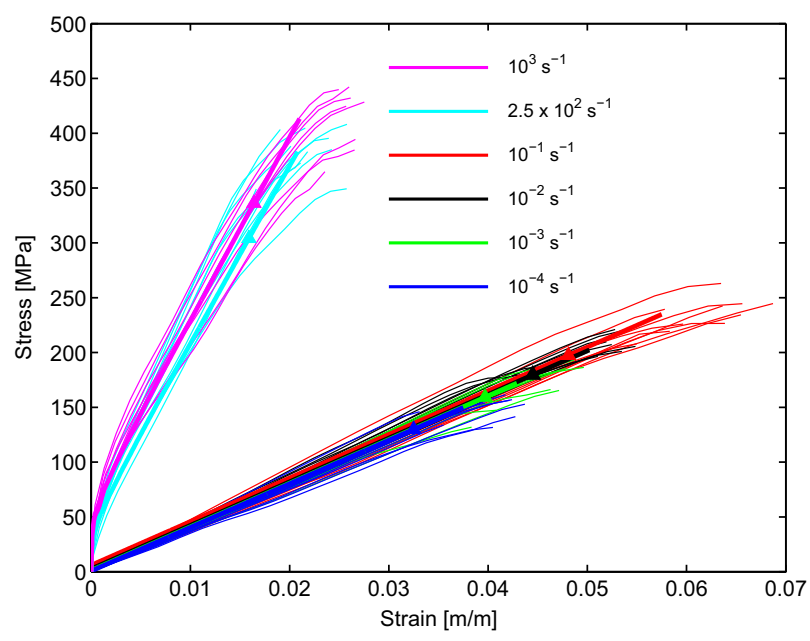

Fig. 7. Average stress-strain curves for quasi-static and dynamic longitudinal compression data. 
Table 1

Ultimate compressive stress $\left(\sigma_{u t}\right)$ and ultimate compressive strain $\left(\varepsilon_{u t}\right)$ for the longitudinal compression of bovine cortical bone at quasi-static and dynamic strain rates. Varying refers to experiments conducted with a uniform area striker. Constant refers to experiments where the input bar pulse was shaped with a tapered striker.

\begin{tabular}{llr}
\hline$\dot{\varepsilon}\left[\mathrm{s}^{-1}\right]$ & $\sigma_{u t}[\mathrm{MPa}]$ & \multicolumn{1}{c}{$\varepsilon_{u t}\left[\mathrm{~m} \cdot \mathrm{m}^{-1}\right]$} \\
\hline$\dot{\varepsilon} \approx 10^{-4}$ & $149.6 \pm 13.7$ & $0.0398 \pm 0.004$ \\
$\dot{\varepsilon} \approx 10^{-3}$ & $171.9 \pm 18.5$ & $0.0451 \pm 0.004$ \\
$\dot{\varepsilon} \approx 10^{-2}$ & $202.1 \pm 14.9$ & $0.051 \pm 0.004$ \\
$\dot{\varepsilon} \approx 10^{-1}$ & $234.8 \pm 15.5$ & $0.062 \pm 0.000$ \\
$\dot{\varepsilon} \approx 2.5 \times 10_{\text {varying }}^{2}$ & $375.7 \pm 30.5$ & $0.024 \pm 0.002$ \\
$\dot{\varepsilon} \approx 2.5 \times 10_{\text {constant }}^{2}$ & $383.9 \pm 21.3$ & $0.024 \pm 0.003$ \\
$\dot{\varepsilon} \approx 1 \times 10_{\text {varying }}^{3}$ & $401.9 \pm 20.6$ & $0.035 \pm 0.003$ \\
$\dot{\varepsilon} \approx 1 \times 10_{\text {constant }}^{3}$ & $413.9 \pm 28.7$ & $0.027 \pm 0.001$ \\
\hline
\end{tabular}

with a dynamic range where it is historically difficult to conduct experiments. The lack of experimental systems and data in the middle strain rate regime is a key factor that prevents the full understanding of the strain rate sensitivity of bone. In fact, it is only recently that Cloete et al. [27] have developed Hopkinson bar techniques to determine the properties of bovine cortical bone in the intermediate strain rate regime.

The implementation of shaped strikers result in a shaped incident pulse to achieve constant strain rate experiments at $\dot{\varepsilon}=2.5 \times 10^{2} \mathrm{~s}^{-1}$ and $\dot{\varepsilon}=10^{3} \mathrm{~s}^{-1}$. Fig. 8a and b shows the incident-, reflected- and transmitted pulses and stress-strain-strain rate responses from a conventional uniform striker experiment. The uniform striker produces the expected trapezoidal incident pulse. It is evident that the corresponding specimen response is not associated with a constant strain rate. In fact, the strain rate drops to approximately $190 \mathrm{~s}^{-1}$ after initially deforming at a rate of nominally $500 \mathrm{~s}^{-1}$. The responses in Fig. $8 \mathrm{c}$ and $\mathrm{d}$ show that the conical striker produces a ramped accelerator pulse with a stress 'tail'. The conical striker succeeded in producing a near constant post ring-up strain rate of approximately $300 \mathrm{~s}^{-1}$. The reflected signals of constant strain rate experiments contain a diagnostic indicator in that a stress plateau is observed in the reflected signal prior to the specimen failure. This is absent in the varying strain rate experiments achieved by a uniform striker. Some challenges of the use of tapered strikers include buckling of the striker tip as a result of excessive stress or bad alignment with the input bar. This resulted in the use of a strengthened steel striker in the higher strain rate experiment (at $10^{3} \mathrm{~s}^{-1}$ ). The alignment of the conical striker in the barrel of the air-gun was improved by sticking light-weight masking tape around a section of the striker with a reduced diameter.

Adharapurapu et al. [17] argued that a variation in strain rate during a test could result in the measurement of smeared properties if the material is strain rate sensitive. This hypothesis was investigated by comparing the results of the constant and varying strain rate experiments. Fig. 9 shows the comparison between constant and varying dynamic strain rate responses at $2.5 \times 10^{2} \mathrm{~s}^{-1}$ and $10^{3} \mathrm{~s}^{-1}$. The strain rate varied between $360 \mathrm{~s}^{-1}$ and $130 \mathrm{~s}^{-1}$ for the experiment at a nominal strain rate of $2.5 \times 10^{2} \mathrm{~s}^{-1}$ test with a uniform striker (Fig. 9b). The results from these experiments are compared to an experiment at a nominally constant strain rate of $\dot{\varepsilon}=2.5 \times 10^{2} \mathrm{~s}^{-1}$. For the experiment at a notional strain rate of $10^{3} \mathrm{~s}^{-1}$ the rate of deformation varied between $1150 \mathrm{~s}^{-1}$ and $780 \mathrm{~s}^{-1}$. The results from this experiment are compared with specimen responses at a strain rate of approximately $10^{3} \mathrm{~s}^{-1}$. a

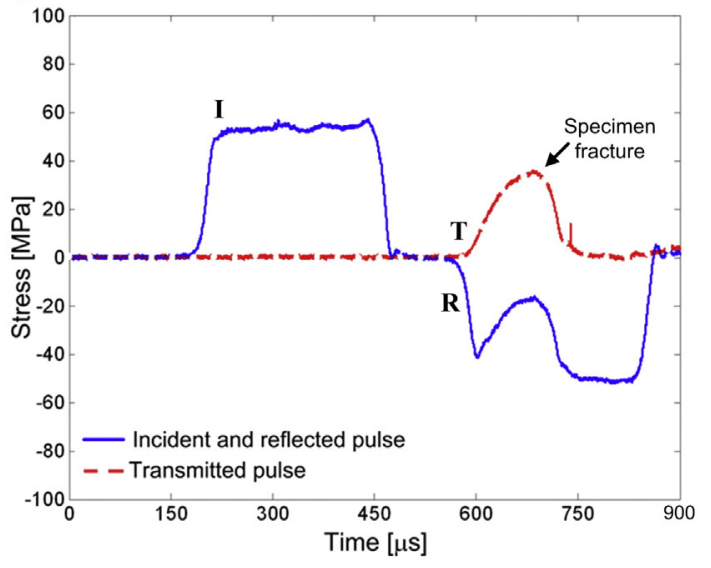

C

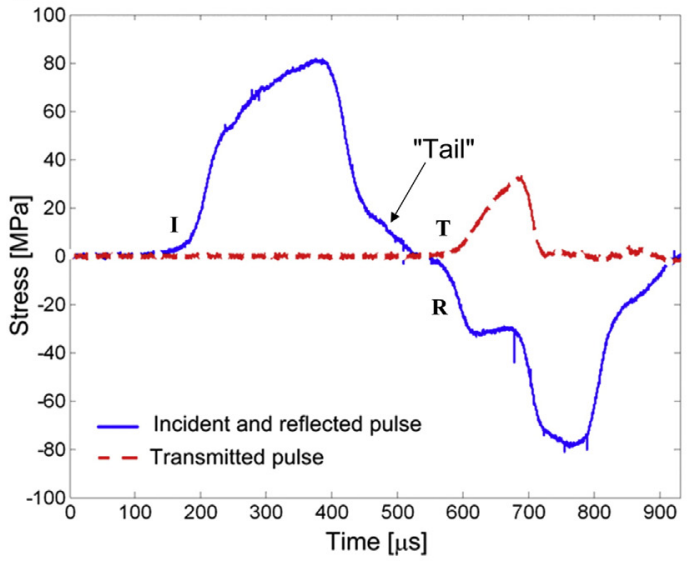

b

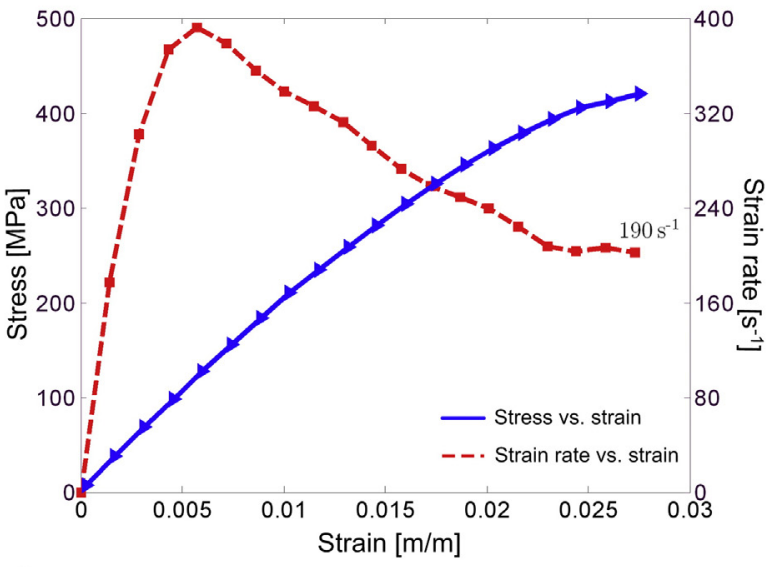

d

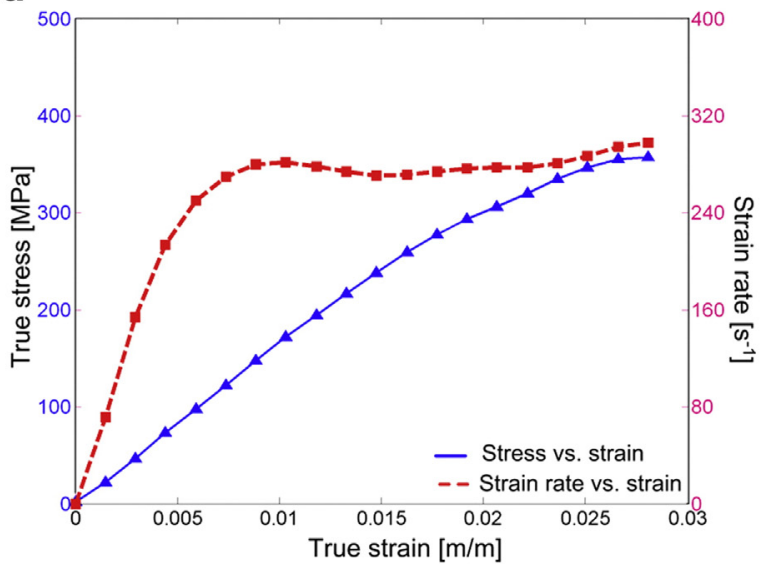

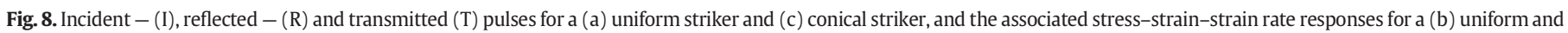
(d) conical striker. 
a

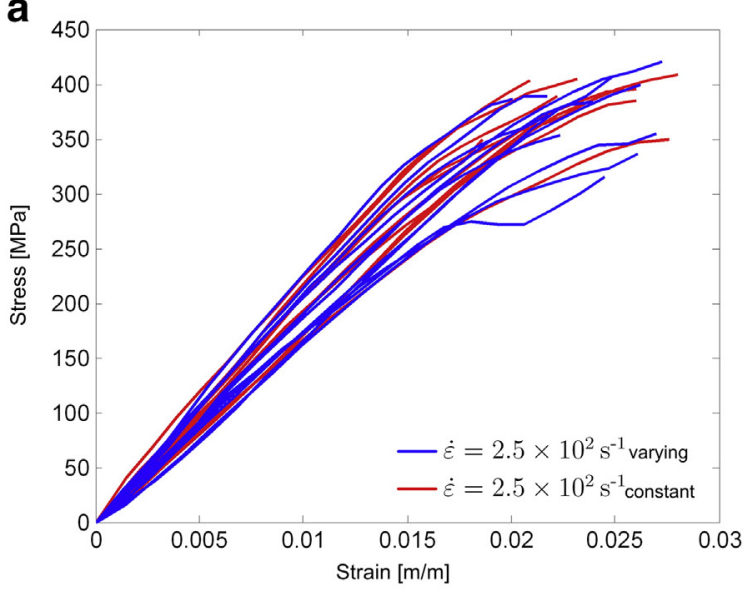

C

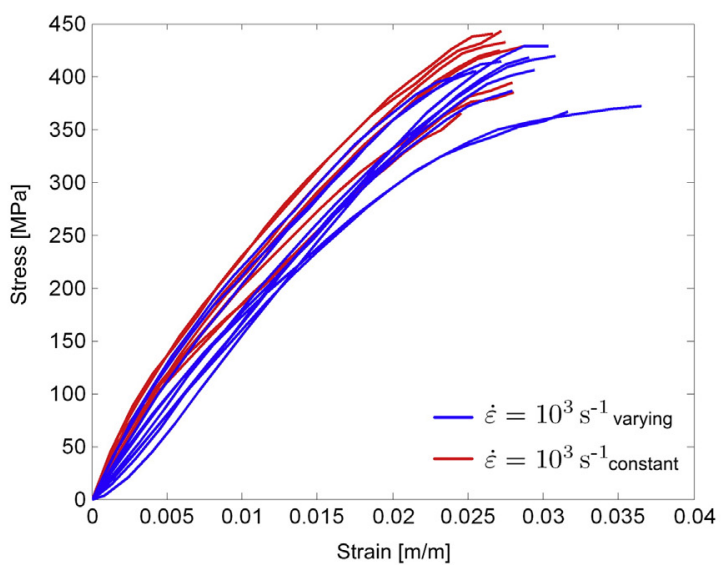

b

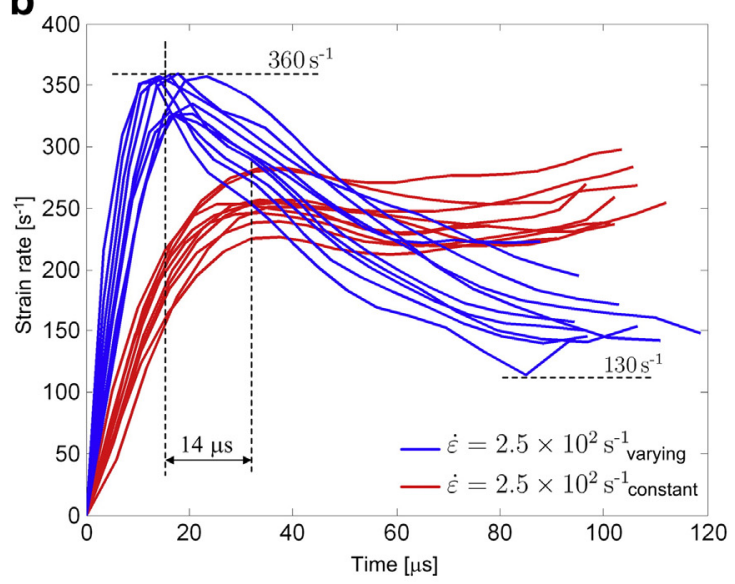

d

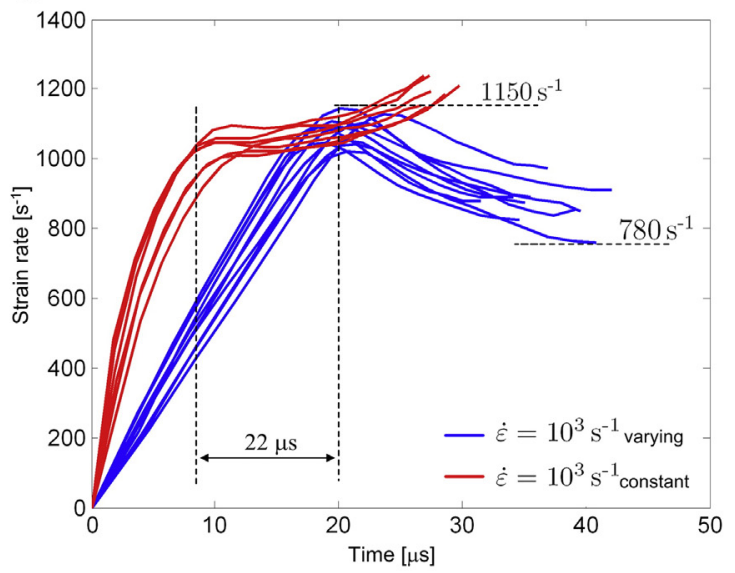

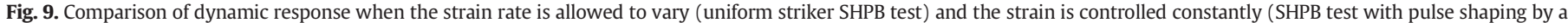
tapered striker) for nominal strain rates of (a, b) $2.5 \times 10^{2} \mathrm{~s}^{-1}$ and (c, d) $10^{3} \mathrm{~s}^{-1}$.

The following observations are made with regard to the effect of strain rate control on the dynamic compressive response of bone: There is uniformity between the response corridors for both of the dynamic strain rates (Fig. 9a and c). At $2.5 \times 10^{2} \mathrm{~s}^{-1}$ the strain rate drops towards the end of the test which causes irregularity in the curvature of the stress-strain curves (Fig. 9a). The average ultimate stress results (Table 1) at both strain rates are slightly lower for tests with a varying strain rate when compared with those where the strain rate was controlled constant. The stresses are $375.7 \mathrm{MPa}$ compared to 383.9 MPa at $2.5 \times 10^{2} \mathrm{~s}^{-1}$ and $401.9 \mathrm{MPa}$ as opposed to $413.9 \mathrm{MPa}$ at $10^{3} \mathrm{~s}^{-1}$. The differences in the ultimate stress at varying strain rates as opposed to constant strain rates are smaller than the standard deviation in the ultimate stress of the respective strain rate regimes. At present it is not possible to conclude if a variation in strain rate during the experiment has a significant influence on specimen ultimate strength.

It is concluded that the tapered striker technique is an advantageous method of pulse shaping because a single tapered striker is effective to control the strain rate of the full series of specimens tested. Thus, inter-specimen variation did not affect the shape of the striker required to achieve a constant strain rate in the present study. Aside from reusability, further advantages of the tapered striker technique include improved test repeatability as the same striker is used in every experiment. Future possibilities include improved control of the pulse shape [22] because of other possible variations in striker geometry.

\section{Discussion and conclusion}

Transverse sections of the bovine femur diaphyses reveal that two arrangements are predominant namely, plexiform microstructure and woven bone. Three microstructural arrangements (laminar, reticular and plexiform) were observed in the longitudinal sections. Bone histology is arranged in roughly concentric rings between the inner and outer bone surfaces in the transverse plane. The vascular porosity of plexiform and laminar cortical bone is approximately aligned with the longitudinal bone axis, whereas the vasculature of reticular arrangements appears unorganised in the bone matrix. It is concluded that the microstructural arrangement in a single bone may vary from location to location and that the effect of varying microstructural arrangements on rate-dependent bone responses is not presently reported for mesoscale models.

Pulse shaping through the method of tapered strikers successfully achieves constant strain rate dynamic compression experiments of bovine cortical bone whilst offering additional advantages in terms of re-usability and test repeatability. The dynamic response corridors of uniform striker - opposed to conical striker SHPB experiments do not differ, however the ultimate properties are slightly affected. Although the experiments from the present study indicate that the dynamic response corridors for constant and varying strain rate experiments are uniform it should be considered that the varying strain rate experiments were carried out in the dynamic regime which displays a low rate sensitivity. The large difference between the high- and low rate regimes suggests high rate sensitivity in the middle strain rate regime. From the results it is recommended that bone compression on the SHPB is conducted with the suitable pulse shaping to achieve a constant strain rate. Additionally it is recommended that the strain rate history should invariably be presented with dynamic stress-strain responses as the instantaneous strain rate is likely a contributor to potential constitutive models. Experimental investigations into bovine bone response in the 
middle strain rate regime $\left(0.1 \mathrm{~s}^{-1}\right.$ to $\left.250 \mathrm{~s}^{-1}\right)$ will greatly contribute to a better understanding of the rate sensitivity of bovine cortical bone.

\section{Acknowledgements}

The contributions of $\mathrm{Mr}$ L. Watkins (Mechanical Workshop, Depart-ment of Mechanical Engineering, University of Cape Town) and Mr. C. Harris (Department of Human Biology, University of Cape Town) are gratefully acknowledged for their respective machining of the strikers and bone specimens.

\section{References}

[1] B. Clarke, Normal bone anatomy and physiology, Clin. J. Am. Soc. Nephrol. 3 (2008) S131-S139.

[2] C.D. Untaroiu, N. Yue, J. Shin, A finite element model of the lower limb for simulating automotive impacts, Ann. Biomed. Eng. 41 (3) (2013) 513-526.

[3] T. Hight, Long-bone criteria for use with the articulated total body model, technical report, Air Force Medical Research Laboratory, Wright-Patterson, 1981.

[4] A. Bekker, S. Kok, T.J. Cloete, G.N. Nurick, Introducing objective power law rate dependence into a visco-elastic material model of bovine cortical bone, Int. J. Impact Eng. 66 (2014) 28-36.

[5] Y. Tanabe, K. Kobayashi, Anisotropy in the dynamic non-linear viscoelastic properties of bovine compact bone, J. Mater. Sci. Mater. Med. 5 (1994) 397-401.

[6] R. Krone, P. Schuster, An Investigation on the Importance of Material Anisotropy in Finite-element Modelling of the Human Femur in SAE International No. 2006-010064, 2006.

[7] P. Lasaygues, M. Pithioux, Ultrasonic characterization of orthotropic elastic bovine bones, Ultrasonics 39 (2002) 567-573.

[8] J.L. Katz, A. Meunier, The elastic anisotropy of bone, J. Biomech. 20 (1987) 1063-1070.

[9] V.Ebacher, C. Tang, H. McKay, T.R. Oxland, P. Guy, R. Wang, Strain redistribution and cracking behavior of human bone during bending. Bone 40 (1) (2007) 1265-1275. [10] P. Zioupos, J.D. Currey, Changes in the stiffness, strength and toughness of cortical bone with age, Bone 22 (1998) 57-66. human

[11] J. Rittweger, G. Beller, J. Ehrig, C. Jung, U. Koch, J. Ramolla, F. Schmidt, D. Newitt

S. Majumar, H. Schiessl, D.Z. Felsenberg, Bone-muscle strength indices for the human lower leg, Bone 27 (2) (2000) 319-326.
[12] R. Dubbeldam, G. Nilson, B. Pal, N. Eriksson, C. Owen, A. Roberts, J. Crandall, I.G. Hal, P. Manning, A. Wallace, A MADYMO of the Foot and Leg for Frontal Impacts in 43rd Stapp Car Crash Conference No. 99SC12, 1999.

[13] J.L. McElhaney, Dynamic response of bone and muscle tissue, J. Appl. Physiol. 21 (1966) 1231-1236.

[14] R.D. Crowninshield, M.H. Pope, The response of compact bone in tension at various strain rates, Ann. Biomed. Eng. 2 (1974) 217-225.

[15] F. Ferreira, M.A. Vaz, J.A. Simoes, Mechanical properties of bovine cortical bone

at high strain rate, Mater. Charact. 57 (2006) 71-79.

[16] R. Tennyson, R. Ewert, V. Niranjan, Dynamic visco-elastic response of bone, Exp. Mech. 12 (1) (1972) 502-507.

[17] R. Adharapurapu, F. Jiang, K. Vecchio, Dynamic fracture of bovine bone, Mater.

Sci. Eng. C 26 (8) (2006) 1325-1332.

[18] U. Hansen, P. Zioupos, R. Currey, J.D. Currey, D. Hynd, The effect of strain rate on

the mechanical properties of human cortical bone, J. Biomech. Eng. 130 (1) (2008) 011011.

[19] H. Kolsky, An investigation of the mechanical properties of materials at very high rates of strain, Proc. R. Phys. Soc. B (1949) 676-700.

[20] J.L. Lewis, W. Goldsmith, The dynamic fracture and prefracture response of compact bone by split Hopkinson bar methods, J. Biomech. 8 (1) (1974) 27-40.

[21] F. Katsamanis, D.D. Raftopoulos, Determination of mechanical properties of human femoral cortical bone by Hopkinson bar stress technique, J. Biomech. 23 (11) (1990) 1173-1184.

[22] A. Kumar, T.S. Lok, Z. Pengjun, Design of an impact striker for a split Hopkinson pressure bar, J. Inst. Eng. Singapore 4 (1) (2004) 119-130.

[23] H. Kobayashi, M. Daimaruya, T. Nojima, T. Kajino, J. Phys. IV 10 (2000) 433-438.

[24] A. Chinsamy, M.A. Raath, Preparation of fossil bone for histological examination, Paideontology Afr. 29 (1992) 39-44.

[25] J.M. Starck, A. Chinsamy, Bone microstructure and developmental plasticity in birds and other dinosaurs, J. Morphol. 254 (2002) 232-246.

[26] N. Sasaki, Y. Nakayama, M. Yoshikawa, A. Enyo, Stress relaxation of bone and bone collagen, J. Biomech. 26 (1) (1993) 1369-1376.

[27] T.J. Cloete, G. Paul, E.B. Ismail, Hopkinson bar techniques for the intermediate strainrate testing of bovine cortical bone, In Proceedings of the Royal Society A, 2014.

[28] M. Kerschnitzki, W. Wagermaier, P. Roschger, J. Seto, R. Shahar, G.N. Duda, S. Mundlos, P. Fratzl, The organization of the osteocyte network mirrors the extracellular matrix orientation in bone, J. Struct. Biol. 173 (2011) 303-311.

[29] J.D. Currey, Bones: Structure and Mechanics, Princeton University Press, 2002.

[30] H.D. Wagner, S. Weiner, On the relationship between the microstructure of bone and its mechanical stiffness, J. Biomech. 25 (11) (1992) 1311-1320. 